\title{
Characterizing and visualizing spatio-temporal patterns in hourly precipitation records
}

Agne Burauskaite-Harju, Anders Grimvall, Alexander Walther, Christine Achberger and
Deliang Chen

\section{Linköping University Post Print}

N.B.: When citing this work, cite the original article.

The original publication is available at www.springerlink.com:

Agne Burauskaite-Harju, Anders Grimvall, Alexander Walther, Christine Achberger and Deliang Chen, Characterizing and visualizing spatio-temporal patterns in hourly precipitation records, 2012, Journal of Theoretical and Applied Climatology, (109), 3-4, 333-343.

http://dx.doi.org/10.1007/s00704-011-0574-X

Copyright: Springer Verlag (Germany) http://www.springerlink.com/?MUD=MP

Postprint available at: Linköping University Electronic Press

http://urn.kb.se/resolve?urn=urn:nbn:se:liu:diva-71297 


\section{Characterizing and visualizing spatio-temporal patterns in hourly precipitation records}

Agne Burauskaite-Harju ${ }^{1 *}$, Anders Grimvall ${ }^{1}$, Alexander Walther ${ }^{2}$, Christine Achberger $^{2}$, Deliang Chen ${ }^{2}$

${ }^{1}$ Linköping University, Linköping, Sweden

${ }^{2}$ Department of Earth Sciences, University of Gothenburg, Sweden

*Corresponding author: Agne Burauskaite-Harju, Division of Statistics, Department of Computer and Information Science, Linköping University, SE-58183 Linköping, Sweden. Phone: +46 13 282785, fax: +46 13 142231, e-mail: agne.burauskaite-harju@liu.se 


\section{ABSTRACT}

Knowledge about spatio-temporal patterns in meteorological data is essential for data assimilation and development of stochastic climate models. Here, we develop new techniques to summarize and visualize spatial patterns of coincidence in weather events such as more or less heavy precipitation at a network of meteorological stations. The cosine similarity measure, which has a simple probabilistic interpretation for vectors of binary data, is generalized to characterize spatial dependencies of events that may reach different stations with a variable time lag. More specifically, we reduce such patterns into three parameters (dominant time lag, maximum cross-similarity, and window-maximum similarity) that can easily be computed for each pair of stations in a network. Furthermore, we visualize such three-parameter summaries by using colour-coded maps of dependencies to a given reference station and distance-decay plots for the entire network. Applications to hourly precipitation data from a network of 93 stations in Sweden illustrate how this method can be used to explore spatial patterns in the temporal synchrony of precipitation events.

KEY WORDS precipitation; hourly rainfall records; spatial dependence; time lag; cosine similarity 


\section{Introduction}

In climate research, considerable attention has long been given to spatio-temporal characteristics of precipitation, temperature, pressure, and other meteorological variables. Knowledge of such features is critical to reveal dynamics of the physical processes responsible for these patterns (e.g., Gong et al., 2007), and also to estimate values for unsampled locations and support assimilation (e.g., Johansson and Chen, 2005). Moreover, it can facilitate development of stochastic climate models and downscaling models of global climate information to regional and local scales (e.g., Haberlandt, 2008; Zheng and Katz, 2008; Baigorria et al., 2007; Brommundt and Bárdossy, 2007; Busuioc et al, 2001; Yang et al., 2005, Buishand and Brandsma, 2001; Wilks, 1998). Here, we focus on the need for new techniques to summarize and visualize spatio-temporal patterns in sub-daily meteorological data, particularly hourly precipitation data from a network of stations.

A common approach to summarizing spatial dependencies in data from a network of stations is to compute the ordinary Pearson correlation for each pair of stations (Gunst, 1995; Robeson and Shein, 1997). Such coefficients can then be plotted against distance between stations to illustrate the distance-decay relationships for precipitation (Osborn and Hulme, 1997), temperature (Jones et al., 1997), surface winds (Achberger et al., 2006), and other meteorological variables. Strong correlations in daily, monthly, and yearly data can persist over relatively large distances, whereas patterns in distance-decay rates for sub-daily data are normally not as clear. Aghakouchak (2010) noted that occurrence of large amounts of hourly precipitation can exhibit strong spatial dependence for sites up to $10 \mathrm{~km}$ apart, whereas other studies have indicated relatively weak dependencies (Serinaldi, 2008; Garcia, 2002; Barbaliscia, 1992).

The low correlation of contemporaneous sub-daily precipitation data from different meteorological stations can often be explained by the fact that synoptic events such as fronts reach the stations with some time lag. Garcia and co-workers (2002) studied crosscorrelations between measurements of hourly precipitation in Spain and demonstrated that the strongest relationship is often found with a time lag that tends to increase with increasing distance. However, the mean time lag being a function of distance alone is rarely so straightforward.

Here, we take the concept of cosine similarity (Tan et al., 2005) as the point of departure for a comprehensive yet simple procedure to describe spatio-temporal patterns in 
sub-daily weather data. This concept is widely used in data mining to quantify the similarity of two vectors, and we apply it to vectors representing the occurrence of extreme precipitation events. In analogy with Pearson's cross-correlation, we also introduce the concept of cross-similarity. Furthermore, we present a procedure in which cross-similarities over a study area are summarized and visualized by introducing three representative parameters: the dominant time lag, the maximum cross-similarity, and a parameter we refer to as the window-maximum similarity.

\section{Methodology}

Our procedure for exploring spatio-temporal characteristics of hourly precipitation data involves three steps. First, cross-similarities are calculated for all pairs of stations. Thereafter, the computed cross-similarities are summarized by computing dominant time lag, maximum cross-similarity, and window-maximum similarity. Finally, the spatial distribution of these parameters is visualized using distance-decay plots and colour-coded maps illustrating the cross-similarities for all pairs, given a reference station.

\subsection{Cosine similarity}

The cosine similarity of two vectors $\boldsymbol{x}=\left(x_{1}, x_{2}, \ldots, x_{n}\right)$ and $\boldsymbol{y}=\left(y_{1}, y_{2}, \ldots, y_{n}\right)$ is defined as

$$
\operatorname{cossim}(\boldsymbol{x}, \boldsymbol{y})=\frac{\sum_{i=1}^{n} x_{i} y_{i}}{\sqrt{\sum_{i=1}^{n} x_{i}^{2} \sum_{i=1}^{n} y_{i}^{2}}}
$$

Geometrically, this measure can be interpreted as the cosine of the angle between $\boldsymbol{x}$ and $\boldsymbol{y}$. Furthermore, it can be noted that, for binary vectors $\boldsymbol{x}^{*}$ and $\boldsymbol{y}^{*}$, the cosine similarity

$$
\operatorname{cossim}\left(\boldsymbol{x}^{*}, \boldsymbol{y}^{*}\right)=\frac{\sum_{i=1}^{n} x_{i}^{*} y_{i}^{*}}{\sqrt{\sum_{i=1}^{n} x_{i}^{*} \sum_{i=1}^{n} y_{i}^{*}}}=\frac{\frac{1}{n} \sum_{i=1}^{n} x_{i}^{*} y_{i}^{*}}{\sqrt{\left(\frac{1}{n} \sum_{i=1}^{n} x_{i}^{*}\right)\left(\frac{1}{n} \sum_{i=1}^{n} y_{i}^{*}\right)}}
$$


is a simple function of three relative frequencies.

Now, let $(\boldsymbol{x}, \boldsymbol{y})$ be a sequence of observations of a bivariate random vector $(X, Y)$ and let $\boldsymbol{x}^{*}$ and $\boldsymbol{y}^{*}$ represent exceedances of a threshold $u$, i.e. set

$$
\begin{aligned}
x_{i}^{*} & =\left\{\begin{array}{l}
1, \text { if } x_{i}>u \\
0, \text { if } x_{i} \leq u
\end{array} \quad i=1,2, \ldots, n\right. \\
y_{i}^{*} & =\left\{\begin{array}{l}
1, \text { if } y_{i}>u \\
0, \text { if } y_{i} \leq u
\end{array} \quad i=1,2, \ldots, n\right.
\end{aligned}
$$

The cosine similarity of $\boldsymbol{x}^{*}$ and $\boldsymbol{y}^{*}$ can then be regarded as an estimate of

$$
\frac{P(\{X>u\} \cap\{Y>u\})}{P(X>u) P(Y>u)}=\sqrt{P(X>u \mid Y>u) P(Y>u \mid X>u)}
$$

i.e., the geometric mean of two conditional probabilities representing the coincidence of two events.

\subsection{Cosine cross-similarity}

The cosine similarity measure of binary data can be generalized to a cosine crosssimilarity function by considering the coincidence of time-lagged events. More specifically, we introduce the function

$$
\operatorname{cossim}\left(\mathbf{x}^{*}, \mathbf{y}^{*}, d\right)=\frac{\sum_{i=\max (1+d, 1)}^{\min (n, n+d)} x_{i}^{*} y_{i-d}^{*}}{\sqrt{\sum_{i=\max (1+d, 1)}^{\min (n, n+d)} x_{i}^{*} \sum_{i=\max (1-d, 1)}^{\min (n, n-d)} y_{i}^{*}}} .
$$

where $d$ is a time lag that can be positive or negative.

\subsection{Maximum cross-similarity and dominant lag}

The maximum cosine cross-similarity

$$
\operatorname{maxsim}\left(\boldsymbol{x}^{*}, \boldsymbol{y}^{*}\right)=\max _{d \in[-(n-1), n-1]} \operatorname{cossim}\left(\mathbf{x}^{*}, \mathbf{y}^{*}, d\right)
$$

of two binary vectors $x^{*}$ and $y^{*}$ is a measure that can be used to describe the strength of the spatial dependence of two types of events, regardless of the time lag at which they occur. In 
formula (4), we consider the maximum over all possible time lags. However, this maximum can also be computed over a user-defined range covering all time lags of practical interest.

The dominant lag

$$
\operatorname{dlag}\left(\boldsymbol{x}^{*}, \boldsymbol{y}^{*}\right)=\underset{d \in[-(n-1), n-1]}{\arg \max }\left(\operatorname{cossim}\left(\mathbf{x}^{*}, \mathbf{y}^{*}, d\right)\right)
$$

is the time lag for which the cross-similarity reaches its maximum. If this lag is not uniquely determined, we set the dominant lag to the smallest lag for which the maximum crosssimilarity is achieved.

\subsection{Window-maximum similarity}

The concept of window-maximum similarity is introduced to measure the coincidence of events that occur with varying time lags. For a given window length $h$ and a pair of binary vectors $\boldsymbol{x}^{*}$ and $\boldsymbol{y}^{*}$, we first construct a new pair of binary vectors $\boldsymbol{x}^{* *}$ and $\boldsymbol{y}^{* *}$ by setting

$$
\begin{aligned}
& x_{i}^{* * *}=\max \left(x_{i}^{*}, x_{i+1}^{*}, \ldots, x_{i+h}^{*}\right), i=1,2, \ldots, n-h \\
& y_{i}^{* * *}=\max \left(y_{i}^{*}, y_{i+1}^{*}, \ldots, y_{i+h}^{*}\right), i=1,2, \ldots, n-h
\end{aligned}
$$

Then we compute the cosine similarity of $\boldsymbol{x}^{* *}$ and $\boldsymbol{y}^{* *}$. This window-maximum similarity is particularly suitable for quantifying the coincidence of events in weather data with high temporal resolution, because the synoptic events under consideration often reach a given pair of stations with varying time lag.

When $(\boldsymbol{x}, \boldsymbol{y})$ is a sequence of observations of a bivariate random vector $(X, Y)$, and $\boldsymbol{x}^{*}$ and $\boldsymbol{y}^{*}$ represent exceedances of a threshold $u$, the window-maximum similarity of $\boldsymbol{x}^{*}$ and $\boldsymbol{y}^{*}$ can be regarded as an estimate of

$$
\frac{P\left(\left\{\max \left(X_{i}, \ldots, X_{i+h}\right)>u\right\} \cap\left\{\max \left(Y_{i}, \ldots, Y_{i+h}\right)>u\right\}\right)}{\sqrt{P\left(\max \left(X_{i}, \ldots, X_{i+h}\right)>u\right) P\left(\max \left(Y_{i}, \ldots, Y_{i+h}\right)>u\right)}}
$$




\subsection{Cosine similarity and Pearson's correlation}

The cosine similarity measure and Pearson's sample correlation coefficient

$$
r(\mathbf{x}, \mathbf{y})=\frac{\sum_{i=1}^{n}\left(x_{i}-\bar{x}\right)\left(y_{i}-\bar{y}\right)}{\sqrt{\sum_{i=1}^{n}\left(x_{i}-\bar{x}\right)^{2} \sum_{i=1}^{n}\left(y_{i}-\bar{y}\right)^{2}}}
$$

for a set of observations of a bivariate random vector $(X, Y)$ are closely related to each other but not identical. More specifically, Pearson's correlation is equal to the cosine similarity computed for data normalized to a mean of zero. Moreover, standard calculations show that, for binary observations representing exceedances of a threshold $u$, Pearson's correlation can be regarded as an estimate of

$$
\frac{\sqrt{P(X>u \mid Y>u) P(Y>u \mid X>u)}-\sqrt{P(X>u) P(Y>u)}}{\sqrt{(1-P(X>u))(1-P(Y>u))}}
$$

Pearson's cross-correlation of a sample of a bivariate random vector $(X, Y)$ is defined as

$$
r(\mathbf{x}, \mathbf{y}, d)=\frac{\sum_{i=\max (1+d, 1)}^{\min (n, n+d)}\left(x_{i}-\bar{x}\right)\left(y_{i-d}-\bar{y}\right)}{\sqrt{\sum_{i=\max (1+d, 1)}^{\min (n, n+d)}\left(x_{i}-\bar{x}\right)^{2}} \sum_{i=\max (1-d, 1)}^{\min (n, n-d)}\left(y_{i}-\bar{y}\right)^{2}}(8)
$$

For binary data representing exceedances of a threshold $u$, this measure is closely related to the previously defined cosine cross-similarity. More explicitly, the two measures satisfy an equation of the form

$$
\operatorname{cossim}\left(\mathbf{x}^{*}, \mathbf{y}^{*}, d\right)=a+b r\left(\mathbf{x}^{*}, \mathbf{y}^{*}, d\right)(9)
$$

where $a$ and $b$ are estimates of $\sqrt{P(X>u) P(Y>u)}$ and $\sqrt{(1-P(X>u))(1-P(Y>u))}$, respectively. This implies that the dominating lag is the same for the cosine cross-similarity and Pearson's cross-correlation. Furthermore, the cross-correlation approaches the crosssimilarity when the threshold $u$ tends to infinity. 


\section{Swedish hourly precipitation data}

Hourly precipitation data from 93 meteorological stations spread throughout Sweden were procured from the Swedish Meteorological and Hydrological Institute (SMHI). The time span of the data was from 1996 to 2008, and we limited our analysis to station series with data covering at least $70 \%$ of this period. The coverage was considerably higher than $70 \%$ for a majority of the selected stations, and the fraction of missing values was less than $10 \%$ for 78 stations. We also noted that the raw data contained a small number of what were most likely unrealistic outliers (> $100 \mathrm{~mm} / \mathrm{h}$ ), which we removed. For a detailed description of the selected data and the initial adjustments that were performed, see Jeong and coworkers (2011) and Wern and German (2006).

\section{Application to the Swedish data}

\subsection{Cross-similarity curves}

Cross-similarity curves were computed for all pairs of the 93 investigated stations. Fig. 1 illustrates those curves for two pairs representing, respectively, two stations far apart $(430 \mathrm{~km})$ and two close to each other $(12 \mathrm{~km})$. In particular, it can be noted that the curves have a single peak and that the cross-similarity decreases with increasing time lag. Visual inspection of cross-similarity curves for a large set of station pairs revealed that almost all pairs exhibited the same characteristics, although the peaks tended to flatten out and become difficult to identify for pairs located farther apart. Moreover, stations close together had cross-similarity peaks at a time lag close to zero, whereas stations far apart exhibited peaks at time lags up to $24 \mathrm{~h}$.

Fig. 1 indicates that, regardless of the spatial distance between investigated stations, the cross-similarity normally decreases with the intensity of the precipitation event. This was confirmed by examining a large number of station pairs. However, as shown in Fig. 1, the cross-similarity can also be substantial for high thresholds, provided the stations are sufficiently close to each other. 
Inspection of the maximum cross-similarity for station pairs belonging to a homogeneous network of stations showed that the spatial pattern of this parameter is similar to that of the maximum Pearson cross-correlation. This is a direct consequence of formula (9) and the fact that the coefficients $a$ and $b$ in this formula represent characteristics of the marginal distributions. For a heterogeneous network, in which the marginal distributions of extreme events may be quite different, the patterns of maximum cross-similarity and crosscorrelation can differ substantially.
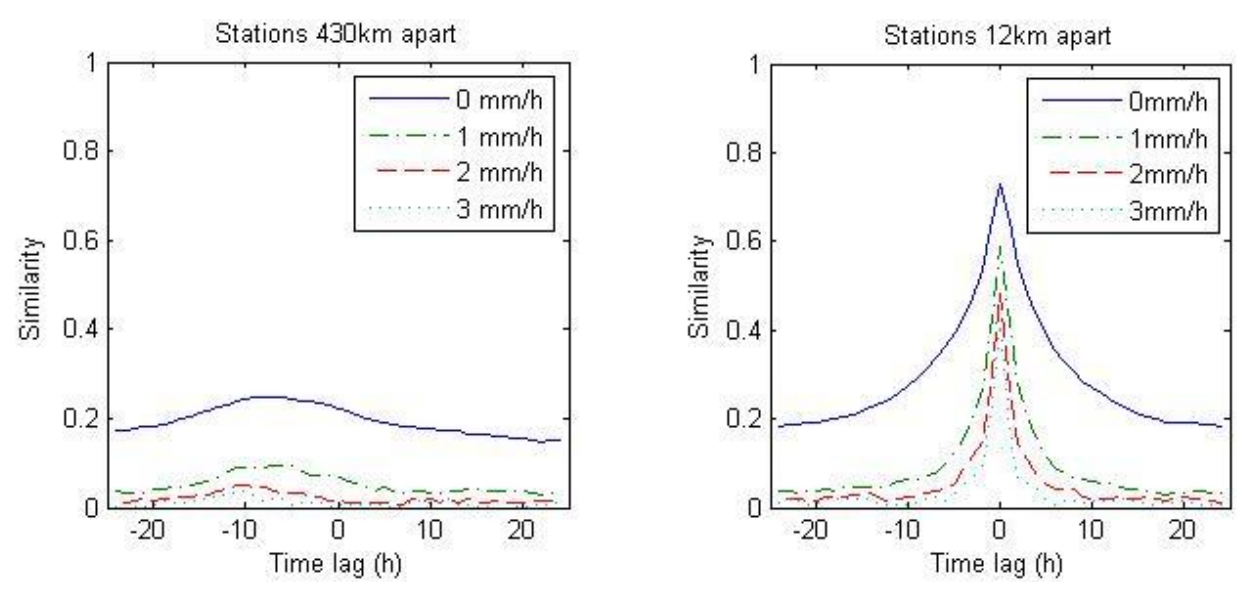

Fig.1 Curves of cosine cross-similarity for one pair of stations located $430 \mathrm{~km}$ (left) apart and another $12 \mathrm{~km}$ apart (right), and four precipitation thresholds $(0,1,2,3 \mathrm{~mm} / \mathrm{h})$

The window-maximum similarity always increases with increasing window length. As shown in Fig. 2, there is also a tendency for this increase to slow down after a certain time lag. This was expected, considering that the spatial dependence will not increase as much when the window already contains the peak of the cross-similarity curve. In our dataset, the dominant time lag normally varied from a few to about 15 hours and was invariably less than 24 hours. This illustrates the general speed at which rain belts move in Sweden. 

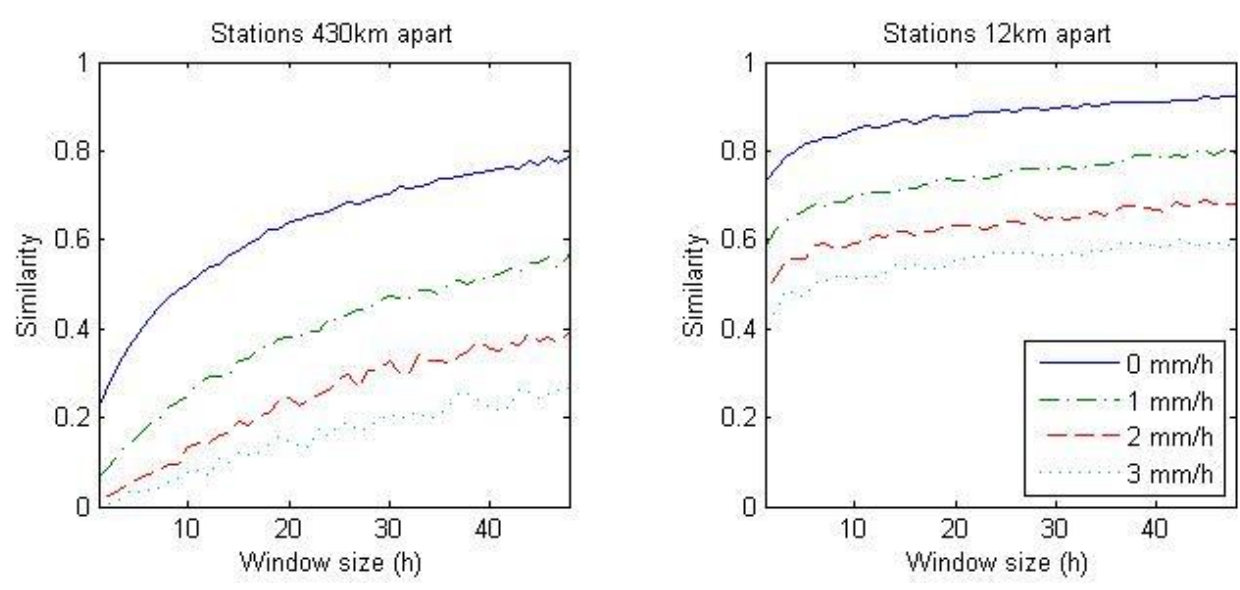

Fig. 2 Window-maximum similarity for the same two pairs of stations and thresholds as shown in Fig.1

\subsection{Summaries of cross-similarities to a reference station}

To be able to summarize cross-similarity data for many pairs of stations, we reduced the cross-similarity curves to the three parameters introduced in section 2: the dominant time lag, the maximum cross-similarity, and the window-maximum similarity. Table 1 shows these parameters for the two pairs of stations illustrated in Fig. 1. Information about dominant time lag, maximum cross-similarity, and window-maximum similarity for all pairs with a fixed reference station is preferably presented as colour-coded maps.

Table 1 Summary of the parameters of the cross-similarity curves computed for the two pairs of stations in Fig. 1

\begin{tabular}{|l|l|l|l|l|l|l|}
\hline \multirow{2}{*}{$\begin{array}{l}\text { Threshold } \\
(\mathrm{mm} / \mathrm{h})\end{array}$} & \multicolumn{2}{|l|}{ Dominant time lag $(\mathrm{h})$} & \multicolumn{2}{l|}{$\begin{array}{l}\text { Maximum } \\
\text { cross-similarity }\end{array}$} & \multicolumn{2}{l|}{$\begin{array}{l}\text { 24-h window-maximum } \\
\text { similarity }\end{array}$} \\
\cline { 2 - 7 } & $430 \mathrm{~km}$ & $12 \mathrm{~km}$ & $430 \mathrm{~km}$ & $12 \mathrm{~km}$ & $430 \mathrm{~km}$ & $12 \mathrm{~km}$ \\
\hline 0 & -7 & 0 & 0.25 & 0.73 & 0.66 & 0.89 \\
\hline 1 & -6 & 0 & 0.10 & 0.59 & 0.43 & 0.74 \\
\hline 2 & -10 & 0 & 0.05 & 0.48 & 0.26 & 0.63 \\
\hline 3 & -10 & 0 & 0.04 & 0.41 & 0.16 & 0.57 \\
\hline
\end{tabular}

Figs. 3 and 4 show maps summarizing the spatial dependence of precipitation events between one reference station in southern Sweden, one in northern Sweden, and all other 
stations. In both figures, the dominant time lag is generally negative for stations located south-west of the reference station and positive for stations north-east of it. Moreover, the dominant time lag gradually changes from about -20 to $20 \mathrm{~h}$, thus illustrating the speed and dominant direction of movement of weather systems in the country. The maps of maximum cross-similarities show the spatial dependence of precipitation events at the dominant time lag. For both reference stations, there is decreasing dependence with increasing separation distance. For hourly precipitation events exceeding $2 \mathrm{~mm}$, the dependence disappears after a short distance, indicating that intense precipitation events occur on a rather local scale.

Repeated visual inspection of a large collection of summary maps of the type shown in Figs. 3 and 4 reveals similar patterns. This means that it is possible to retrieve the main characteristics of spatial dependencies by exploring a small number of summary maps.
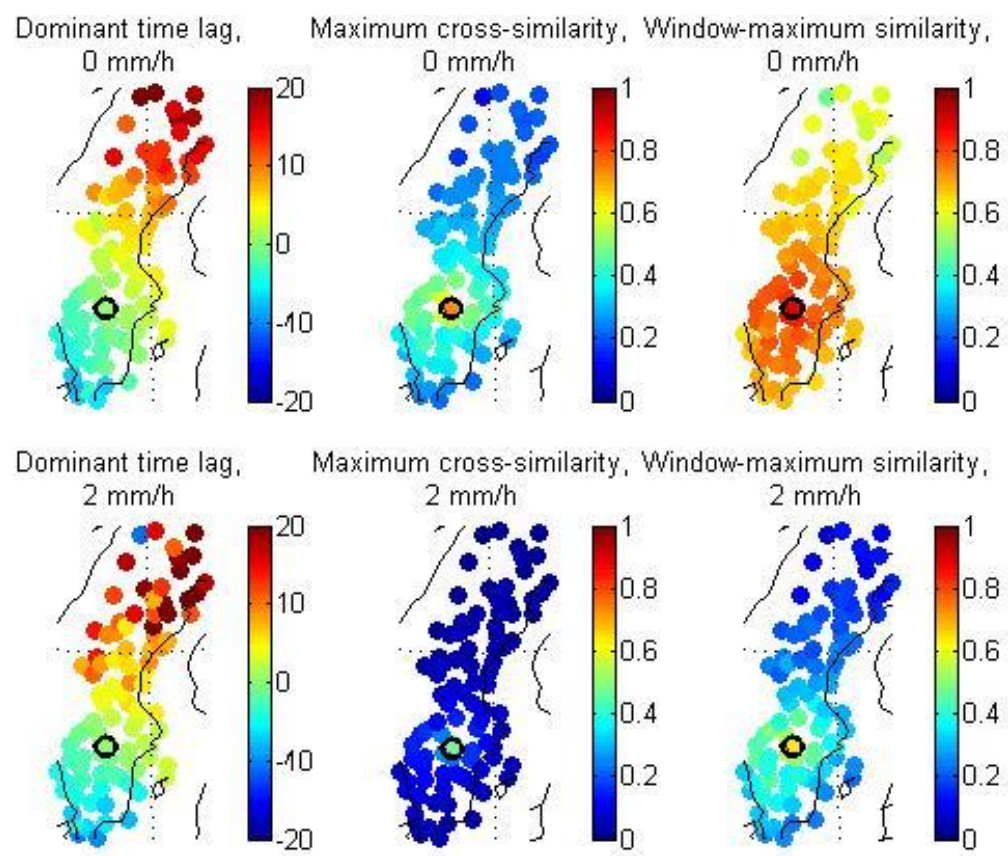

Fig. 3 Summary maps representing dominant time lag, maximum cross-similarity, and 24-h windowmaximum similarity for hourly precipitation data from all pairs of stations in relation to a reference station in Southern Sweden (black circle). Thresholds of precipitation events were set to 0 and $2 \mathrm{~mm} / \mathrm{h}$, respectively 


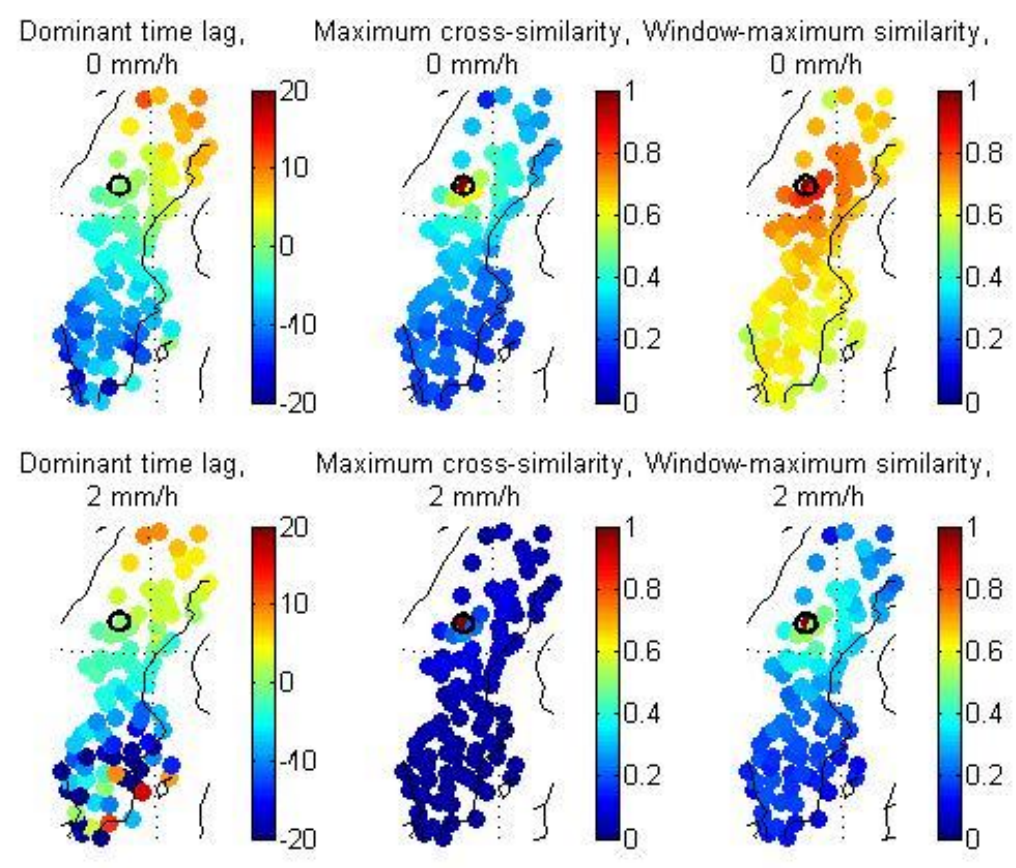

Fig. 4 Summary maps representing dominant time lag, maximum cross-similarity, and 24-h windowmaximum similarity for hourly precipitation data from all pairs of stations in relation to a reference station in Northern Sweden (black circle). Thresholds of precipitation events were set to 0 and $2 \mathrm{~mm} / \mathrm{h}$, respectively

\subsection{Summary of cross-similarities for a network of stations}

In order to explore the spatial dependence of precipitation events for all pairs of stations in a given network, we plotted the three cross-similarity parameters introduced in section 2 against distance. As expected, the absolute value of the dominant time lag increased with the distance to the reference station. However, there were also other spatio-temporal patterns of interest. Fig. 5 shows that the markers of the investigated pairs of stations are concentrated along a straight line with a slope of $2.5 \mathrm{~h} / 100 \mathrm{~km}$ for precipitation amounts exceeding $2 \mathrm{~mm}$, and a slope of $2 \mathrm{~h} / 100 \mathrm{~km}$ when considering all precipitation events. In addition, very few markers are above the lines representing slopes of 3.5 and $2.5 \mathrm{~h} / 100 \mathrm{~km}$, respectively. The higher slope for larger amounts of precipitation indicates that such events are associated with weather systems such as fronts moving relatively slowly across the study area.

Typical relationships between window-maximum similarities and the distance between stations are illustrated in Fig. 6. It seems that these measures of spatial dependence normally decrease with distance but remain clearly above zero even for stations that are far 
apart. Furthermore, it can be noted that the subsets of markers representing different window lengths are generally well separated. Closer examination of deviating pairs of stations reveals that they represent highland areas, where the topographic conditions can distort overall spatial dependencies.

The maps in Figs. 3 and 4 indicate that the window-maximum similarity is significantly greater than the maximum cross-similarity. Fig. 6 shows more generally how this similarity measure varies with distance between stations, window length, and intensity of the precipitation event under consideration. The difference in distance-decay relationships between the two precipitation amounts can be explained by the fact that heavy precipitation events typically occur in relatively small areas.
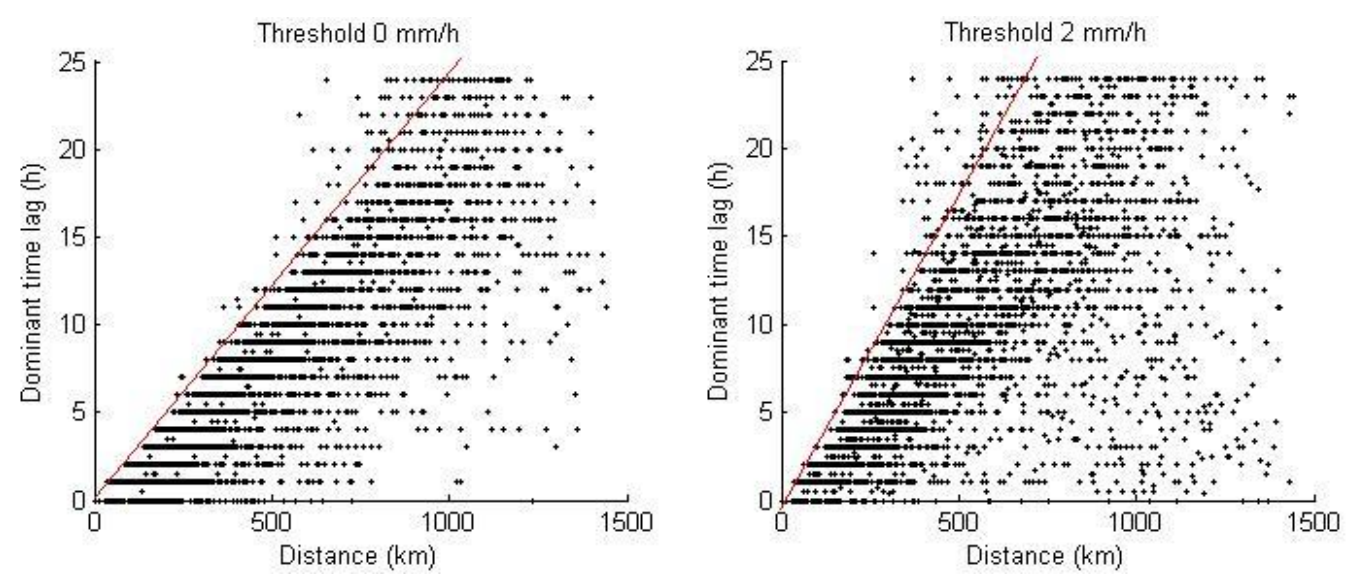

Fig. 5 Relationship between dominant time lag and separation distance for all investigated pairs of stations and two precipitation thresholds ( 0 and $2 \mathrm{~mm} / \mathrm{h})$
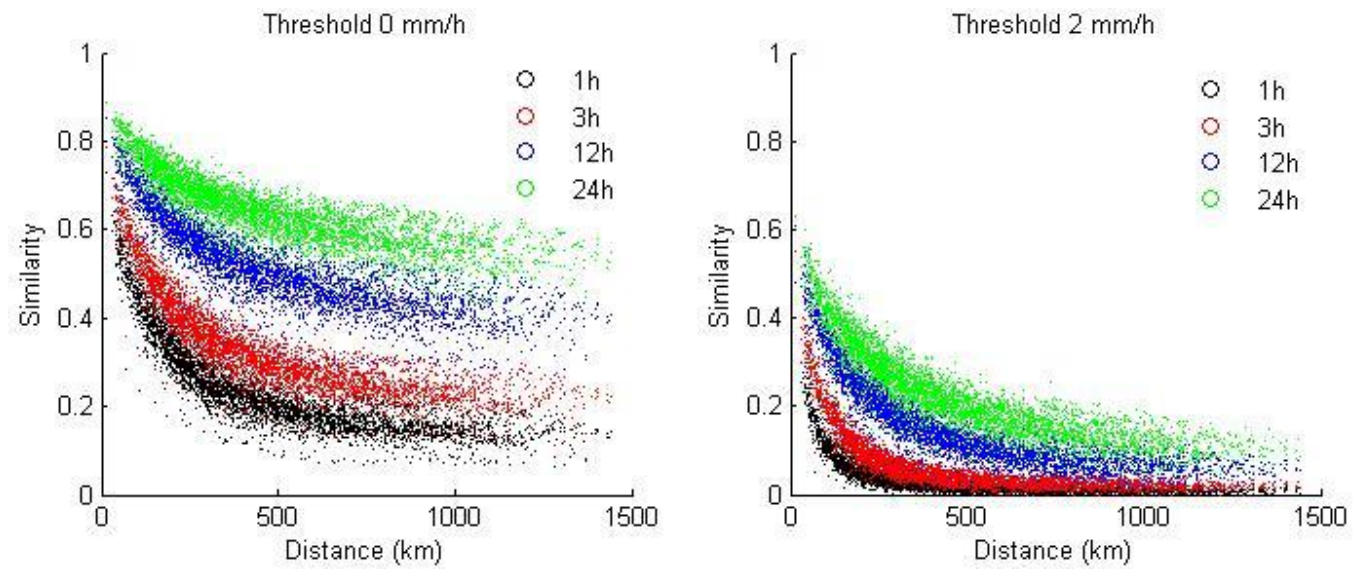

Fig. 6 Distance-decay relationships for window-maximum similarities computed for four window lengths $(1,3,12$, and $24 \mathrm{~h})$ and two precipitation thresholds $(0$ and $2 \mathrm{~mm} / \mathrm{h})$ 


\subsection{Seasonal patterns of cross-similarity}

The similarity measures introduced in section 2 can be used to reveal seasonal patterns of spatio-temporal dependencies. We investigated the presence of those patterns in summer (April to September, AMJJAS) and winter (October to March, ONDJFM) separately.

When the threshold was set to zero, we noted a clear summer-winter difference in the window-maximum similarities and maximum cross-similarities. This is illustrated in Fig. 7, which shows similarities between a reference station in southern Sweden and all other stations. Furthermore, we observed that the dominant time lags increased more rapidly with distance in summer than in winter. Closer examination of the dominant time lags indicates that movement of weather systems from the south-west towards the north-east is the main pattern in both seasons. However, there is a noticeable difference for a group of stations in western Sweden. The results obtained for a precipitation threshold of $2 \mathrm{~mm} / \mathrm{h}$ are more uncertain due to the relatively small number of those events. In particular, our findings show that the dominant time lag can vary significantly for stations located far apart. 
a)

Summer (AMJJAS)

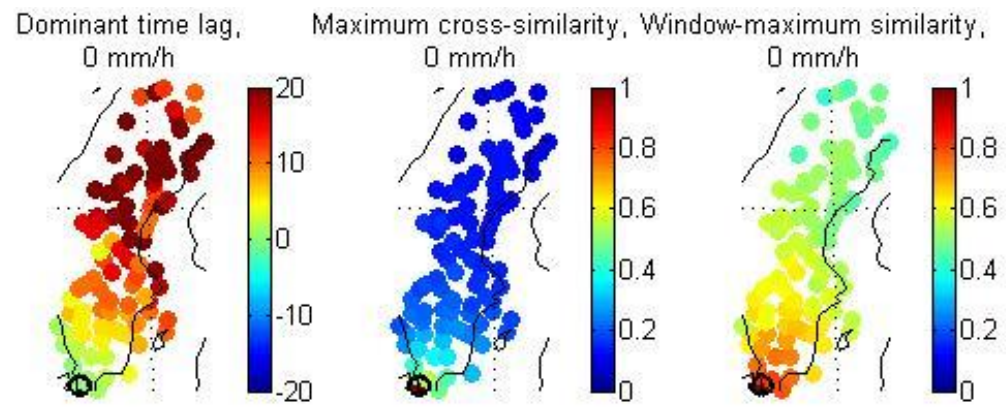

Dominant time lag, Maximum cross-similarity, Window-maximum similarity,

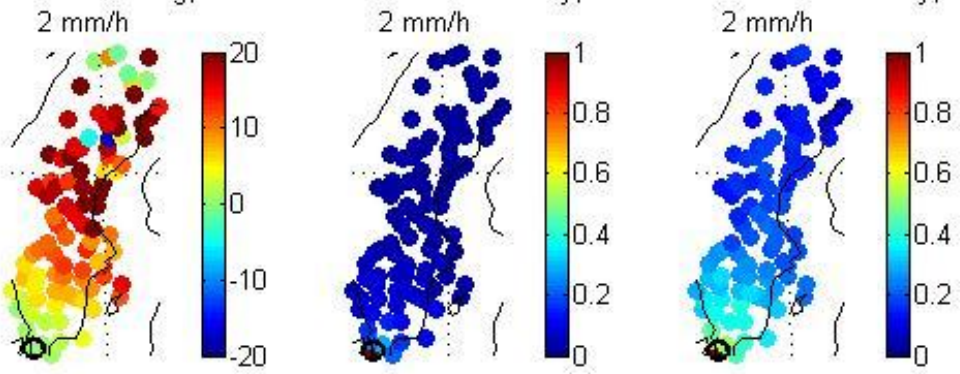

b)

Winter (ONDJFM)

Dominant time lag, Maximum cross-similarity, Window-maximum similarity,

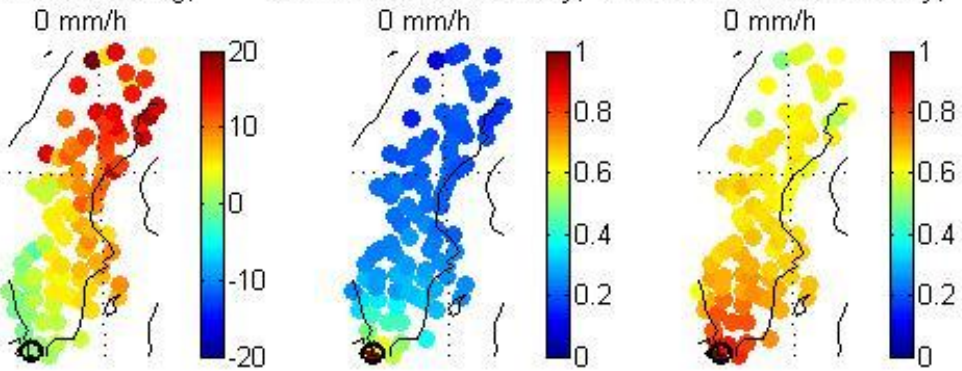

Dominant time lag, Maximum cross-similarity, Window-maximum similarity,

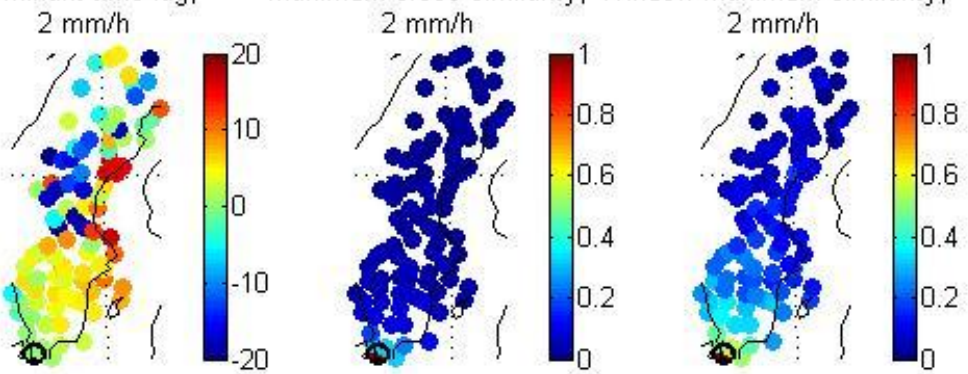

Fig. 7 Summary maps of dominant time lag, maximum cross-similarity, and window-maximum similarity for summer (a) and winter (b) and two precipitation thresholds (0 and $2 \mathrm{~mm} / \mathrm{h}$ ). The reference station in southern Sweden (black circle) is the same in all maps 
The plots shown in Fig. 8 show that the dominant lag increases more rapidly in summer than in winter in relation to distance between stations. This indicates that weather systems move more slowly and/or take irregular pathways in summer. Furthermore, it is worth noting that, regardless of the threshold, precipitation events exhibit a wider range of dominant time lags in winter.
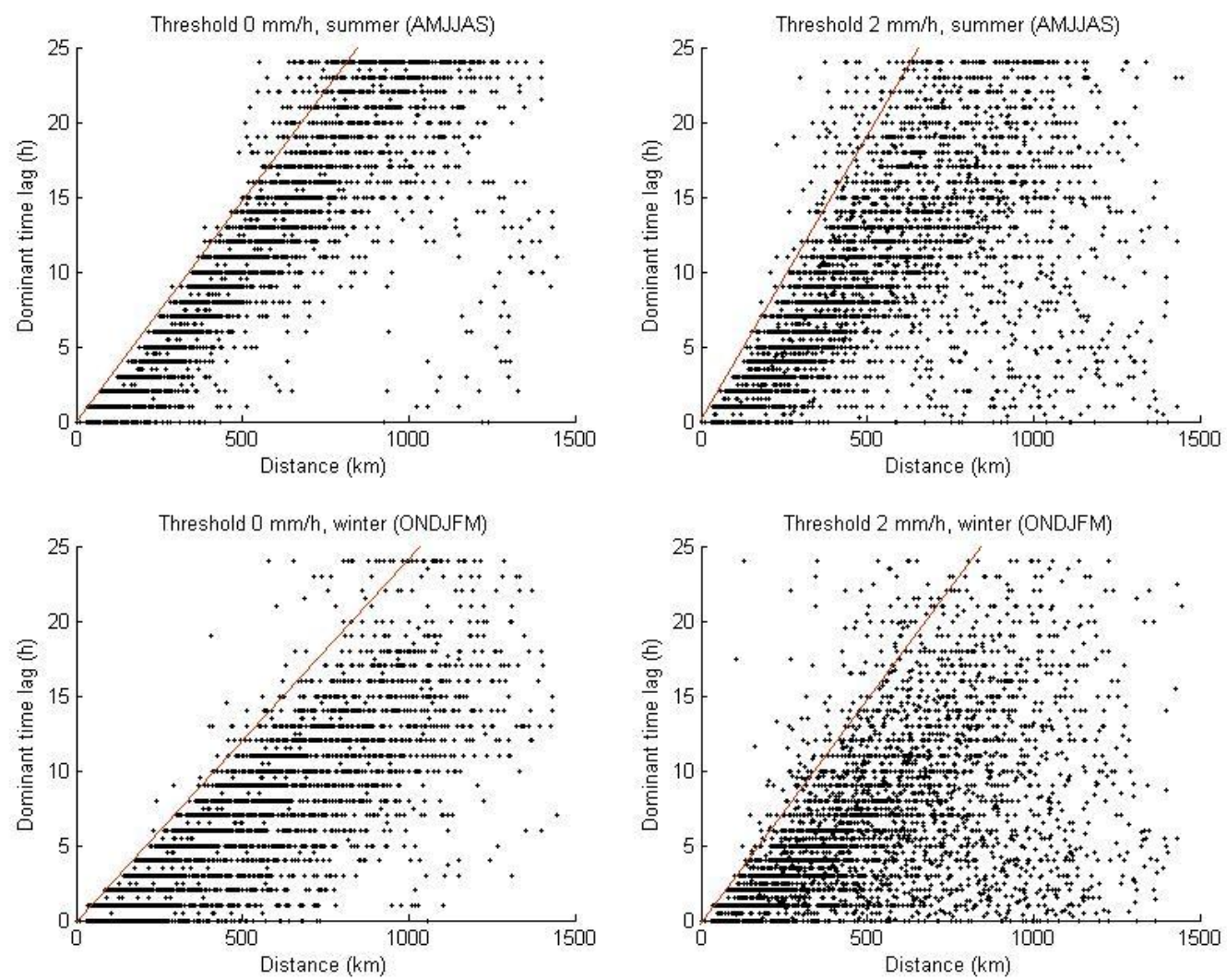

Fig. 8 Relationship between dominant time lag and station separation distance and two precipitation thresholds $0 \mathrm{~mm} / \mathrm{h}$ (left) and $2 \mathrm{~mm} / \mathrm{h}$ (right) in summer and winter

Fig. 9 shows that the distance-decay relationships are similar in summer and winter. However, the window-maximum similarity is larger in summer for distances exceeding 500 $\mathrm{km}$. In addition, it is apparent that the relationships are not as clear in winter, partly due to the small number of heavy precipitation events during this season. 

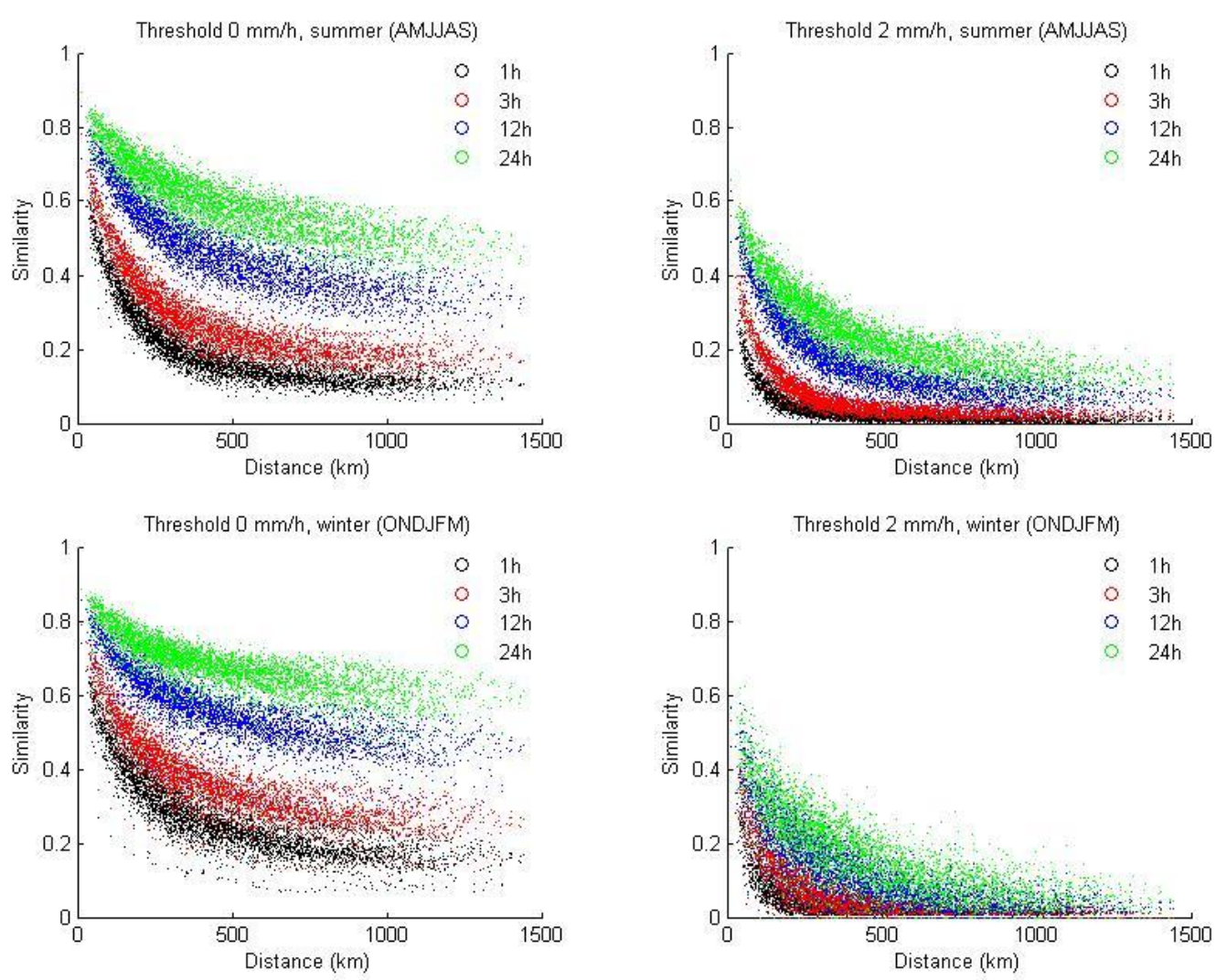

Fig. 9 Distance-decay relationships in winter and summer for window-maximum similarities computed for four window lengths $(1,3,12$, and $24 \mathrm{~h})$ and two precipitation thresholds $(0$ and $2 \mathrm{~mm} / \mathrm{h})$

\section{Discussion and conclusions}

Hourly precipitation records for a network of meteorological stations can exhibit very complex spatio-temporal patterns. Here, we have presented a procedure that enables efficient exploration of the coincidence of precipitation events of varying intensity in a network of stations. In particular, we have shown how spatial dependencies in temporally synchronized precipitation events can be effectively summarized and visualized.

Cosine similarity is a measure of dependence that is widely used in data mining, especially text mining (Tan et al., 2005), but is applied to a much lesser extent in geosciences, where correlograms and variograms are the key concepts for spatio-temporal modelling (Diggle \& Ribeiro, 2007; Chen et al., 2007). We chose cosine similarity as the point of departure in our study for two reasons: (i) we were interested in binary data representing the occurrence of specific weather events; (ii) in contrast to Pearson's correlation, cosine similarity has a simple probabilistic interpretation when the underlying 
data are binary time series. Accordingly, the generalization to cross-similarities of events recorded at two stations followed the same line as the generalization of correlation to crosscorrelation. By further generalizing the cross-similarity to window-maximum similarity, we were able to handle the fact that a certain type of event can appear at two stations with a variable time lag.

From a computational point of view, it is easy to produce a large number of crosssimilarity functions. However, interpretation of the results that are obtained can be greatly facilitated by reducing the spatio-temporal patterns to a small number of characteristics for each station pair. Therefore, we introduced a three-parameter summary comprising dominant time lag, maximum cross-similarity, and window-maximum similarity. Furthermore, we proposed colour-coded maps and distance-decay plots to visualize the information in these summaries. The colour-coded maps are particularly useful to investigate anisotropy and how similarities vary in relation to the location of the reference station. The distance-decay plots offer the benefit of providing a comprehensive overview of spatial dependencies for the entire network.

By applying our technique to hourly precipitation records acquired from a network of stations in Sweden, we found that this procedure can reveal important characteristics of the spatial and temporal coincidence of precipitation events. The colour-coded maps of dominant time lags in Figs. 3 and 4 show how precipitation-generating weather systems normally move across Sweden, driven primarily by the prevailing south-westerly winds. In addition, the distance-decay plots in Fig. 6 reveal that the spatial dependence of heavy precipitation events was significant up to a distance of about $200 \mathrm{~km}$ between stations, although it was considerably weaker than that of precipitation events in general. Our examination of seasonal patterns in the dependence structure demonstrated that the methods we have proposed can enable a rapid comparison of spatio-temporal features of different subsets of binary data.

This study was devoted solely to observational precipitation data. However, the methods can also be used to support development, improvement, and validation of highresolution precipitation models (Haberlandt, 2008), as well as regional climate models such as the RCA3 run by the Rossby Centre at the Swedish Meteorological and Hydrological Institute (SMHI). In its simplest form, the modelling support may be confined to visual comparison of three-parameter summaries of observational data and model outputs. However, if an objective function is used to optimize inter-site dependence in model outputs 
(Haberlandt, 2008), parameters like window-maximum similarity can be incorporated into that function.

\section{Acknowledgments}

The authors are very grateful to the Swedish Meteorological and Hydrological Institute (SMHI) for providing the precipitation data, to Colin Jones at the Rossby Centre for valuable comments and discussions, and to the Swedish Research Council (VR), the Gothenburg Atmospheric Science Centre (GAC), and FORMAS (grant \#2007-1048-8700*51) for financial support to Deliang Chen and Alexander Walther.

\section{References}

Achberger C, Chen D, Alexandersson H (2006) The surface winds of Sweden during 1999-2000. Int J Climatol 26:159-178

Aghakouchak A, Ciach G, Habib E (2010) Estimation of tail dependence coefficient in rainfall accumulation fields. Advances in Water Resources 33 (9):1142-1149. doi:10.1016/j.advwatres.2010.07.003

Baigorria GA, Jones JW, O'Brien JJ (2007) Understanding rainfall spatial variability in southeast USA at different timescales. International Journal of Climatology 27 (6):749-760. doi:10.1002/joc.1435

Barbaliscia F, Ravaioli G, Paraboni A (1992) Characteristics of the spatial statistical dependence of rainfall rate over large areas. IEEE Transactions on Antennas and Propagation 40 (1):8-12

Brommundt J, Bárdossy A (2007) Spatial correlation of radar and gauge precipitation data in high temporal resolution. Adv Geosci 10:103-109. doi:10.5194/adgeo-10-103-2007

Buishand TA, Brandsma T (2001) Multisite simulation of daily precipitation and temperature in the Rhine basin by nearest-neighbor resampling. Water Resources Research 37 (11):2761-2776

Busuioc A, Chen D, and Hellström C (2001) Temporal and spatial variability of precipitation in Sweden and its link with the large scale atmospheric circulation. Tellus 53A (3): 348-367.

Chen D, Gong L, Xu C, Halldin S (2007) A high-resolution, gridded dataset for monthly temperature normals (1971-2000) in Sweden. Geografiska Annaler. 89A(4): 249-261.

Diggle P, Ribeiro PJ (2007) Model-based geostatistics. Springer, 228 p. 
Garcia P, Zambudio N, Benarroch A (2002) Joint Rainfall Rate statistics for Pairs of Sites in Spanish Regions. COST action 280 "Propagation Impairment Mitigation for Millimetre Wave Radio Systems", 1st International Workshop

Gong, DY, Ho CH, Chen D, Qian Y, Choi, YS and Kim J (2007) Weekly cycle of aerosol-meteorology interaction over China. Journal of Geophysical Research 112 (D22202): doi:10.1029/2007JD008888.

Gunst RF (1995) Estimating spatial correlations from spatial-temporal meteorological data. Journal of Climate 8 (10):2454-2470

Haberlandt U, Ebner von Eschenbach AD, Buchwald I (2008) A space-time hybrid hourly rainfall model for derived flood frequency analysis. Hydrol Earth Syst Sci 12 (6):1353-1367. doi:10.5194/hess-12-13532008

Jeong J-H, Walther A, Nikulin G, Chen D, Jones C (2011) Diurnal cycle of precipitation amount and frequency in Sweden: observation versus model simulation. Tellus A. doi:10.1111/j.1600-0870.2011.00517.x

Johansson B and Chen D (2005) Estimation of areal precipitation for runoff modelling using wind data: A case study in Sweden. Climate Research 29: 53-61.

Jones PD, Osborn TJ, Briffa KR (1997) Estimating sampling errors in large-scale temperature averages. Journal of Climate 10 (10):2548-2568

Osborn TJ, Hulme M (1997) Development of a relationship between station and grid-box rainday frequencies for climate model evaluation. Journal of Climate 10 (8):1885-1908

Robeson SM, Shein KA (1997) Spatial coherence and decay of wind speed and power in the northcentral United States. Physical Geography 18 (6):479-495

Serinaldi F (2008) Analysis of inter-gauge dependence by Kendall's tau(K), upper tail dependence coefficient, and 2-copulas with application to rainfall fields. Stochastic Environmental Research and Risk Assessment 22 (6):671-688. doi:10.1007/s00477-007-0176-4

Tan PN, Steinbach M, Kumar V (2006) Introduction to data mining. Pearson Addison Wesley, 769p.

Wern L, German J (2009) Korttidsnederbörd i Sverige 1995 - 2008. Meteorologi, 139/2009. SMHI.

Wilks DS (1998) Multisite generalization of a daily stochastic precipitation generation model. Journal of Hydrology 210 (1-4):178-191

Yang C, Chandler RE, Isham VS, Wheater HS (2005) Spatial-temporal rainfall simulation using generalized linear models. Water Resources Research 41 (11). doi:W1141510.1029/2004wr003739 
Zheng XG, Katz RW (2008) Simulation of spatial dependence in daily rainfall using multisite generators. Water Resources Research 44 (9). doi:W0940310.1029/2007wr006399 